\title{
Beyond the first trimester screen: Can we predict who will choose invasive testing?
}

\author{
Suzanne van Landingham, $A B^{I}$, Jessica Bienstock, $M D, M P H^{1}$, Elizabeth Wood Denne, $M S^{2}$, \\ and Nancy Hueppchen, MD, MSc ${ }^{1}$
}

\begin{abstract}
Purpose: The purpose of this study is to determine what factors, in addition to a positive first trimester aneuploidy screen, correlate with a pregnant patient's decision to undergo invasive prenatal testing. Methods: We conducted a retrospective cohort study of singleton pregnancies referred to the Johns Hopkins Prenatal Diagnosis and Treatment Center between 2001 and 2009 with an indication of positive first trimester screen. We compared demographic factors and numerical first trimester screen results with invasive testing uptake. Risk difference calculations and linear modeling were used for analysis. Results: A total of 171 eligible patients were identified. Maternal age, race, residual risk, marital status, and year of first trimester screen correlated significantly with invasive testing uptake. Family history was predictive of invasive testing uptake for patients younger than 35 years only. Type of elevated risk (trisomy 21 vs. 18 and 13), assisted reproductive technology status, parity, and increase from age-related risk were not predictive. A general linear model for relative risk with Gaussian error showed significant interaction between the variables of age and family history, so the two traits were analyzed separately $(P=0.009)$. Conclusions: Among patients with positive first trimester screen results, several demographic traits are predictive of invasive testing uptake. This information can help providers to identify patients at increased risk of declining invasive testing and can help providers anticipate educational needs. Further investigation should be conducted to elucidate the causes of these differences, which may relate to misinformation about the testing options and differences in values systems. Genet Med 2011:13(6):539-544.
\end{abstract}

Key Words: prenatal diagnosis, first trimester screen, aneuploidy, invasive testing, genetic counseling

$P$ roviding pregnant patients with an accurate, low-risk assessment of fetal chromosome abnormalities has long been a goal in prenatal diagnostics. Chorionic villus sampling (CVS), performed at 11-13 weeks, and amniocentesis, performed at 16-22 weeks, use karyotyping to detect fetal aneuploidy with a sensitivity of $>99 \% .{ }^{1}$ These invasive tests are considered diagnostic. The risk of miscarriage after each test is $<1 \%$ but can be a source of anxiety for patients. ${ }^{2}$

Several noninvasive screening tests are available to assess patients' probability of fetal aneuploidy before exposing them to the risks of diagnostic testing. The second trimester maternal serum screen, developed in the late 1980s, is useful for patients

From the ${ }^{1}$ Johns Hopkins University School of Medicine; ${ }^{2}$ Departments of Obstetrics and Gynecology and Prenatal Diagnostic Treatment Center, Johns Hopkins Medicine, Baltimore, Maryland

Suzanne van Landingham, Johns Hopkins Hospital, Doctors' Lounge, 110 Harvey/Nelson Building, 111 W. Centre Street, Baltimore, MD 21201. E-mail: swestbr3@jhmi.edu.

Disclosure: The authors declare no conflict of interest.

Submitted for publication October 13, 2010

Accepted for publication December 7, 2010.

Published online ahead of print March 17, 2011

DOI: 10.1097/GIM.0b013e31820a7701 considering amniocentesis and has dramatically reduced the uptake of invasive testing (IT) over time. ${ }^{3}$ The more recent introduction of the first trimester screen (FTS) in the early 2000s assesses patients' risk during the 11th-13th week of pregnancy and is, therefore, useful for patients considering CVS or amniocentesis. It provides a probability that a fetus has one of the three most common trisomies, 21,18 , or 13 , with a sensitivity of $>95 \%{ }^{4}$

The purpose of the FTS is to help patients make informed decisions regarding IT. When the screen was first introduced, it was thought that all patients with positive screen results would pursue CVS or amniocentesis. Nicolaides et $\mathrm{al}^{5}$ states that "in prenatal screening for trisomy 21 , the term screen positive rate is used interchangeably with the invasive testing rate, because most women with a positive screening test undergo an invasive test..." This statement reflects what was a commonly held opinion during the early days of FTS. Since that time, however, this idea has been challenged. Studies show IT uptake rates of 33-90\% after positive FTS, indicating that factors other than positive FTS results play into the decision of IT uptake. ${ }^{2}$

Prior research suggests that decision making about IT is not systematic and that patients are often poorly informed. ${ }^{6-8}$ Marteau et al., ${ }^{9}$ for instance, have developed a tool called the "Multidimensional Measure of Informed Choice" that consists of an eight-item scale of knowledge and a four-item scale of attitudes toward prenatal screening. This scale has been used to assess informed decision making regarding prenatal aneuploidy screening in nations as diverse as the United Kingdom, the Netherlands, Greece, and Australia. Each study revealed considerable knowledge deficits, with informed decision-making rates varying from 5 to $71 \%$ across the different groups. ${ }^{6,10,11}$

Other studies have sought to identify particular patient characteristics associated with higher rates of IT uptake. Most of these studies concern the second trimester screen, which is older and more widely available than the FTS. Characteristics associated with lower IT uptake include lower abnormal residual risk and low socioeconomic status. ${ }^{12-14}$ Data are mixed regarding IT uptake in older mothers and couples who required assisted reproduction. ${ }^{12-19}$ This variety may reflect different study methodologies or populations. The studies cited were conducted in Turkey, Lebanon, Canada, the United Kingdom, the United States, and Greece, representing a broad range of health care systems and cultural milieus.

Several studies have shown that the rate of IT uptake has continued to drop after the introduction of the FTS. ${ }^{13,20}$ This trend may reflect many different factors, including increasingly effective use of the prenatal screening or changing perceptions of IT. Given the growing popularity and availability of this screening option, it is important to further study its role in IT uptake.

In this study, we sought to determine what demographic and historical factors, in addition to a positive first trimester aneuploidy screen, correlate with a pregnant patient's decision to undergo IT. We suspected that older mothers would be more 
likely to select IT, given clinical experience and that the increase in the risk of fetal aneuploidy with increasing maternal age is widely known. Elucidation of these factors may reveal disparities in IT uptake and help clinicians to better anticipate patients' educational needs.

\section{MATERIALS AND METHODS}

\section{Subjects}

Demographic and historical data were collected from the records of singleton pregnancies referred to the Johns Hopkins Prenatal Diagnosis and Treatment Center (JHPDC) for genetic counseling between 2001 and 2009 with an indication of positive FTS results. The FTS was administered by the referring provider. At the JHPDC, each patient had an approximately 1-hour long session with a certified genetic counselor and received information about aneuploidy, FTS, and IT. Exclusion criteria included miscarriage or loss to follow-up before the opportunity for IT. Patients with other indications for IT, such as family history of other genetic disorders, were also excluded. A total of 171 patients of approximately 200 with abnormal FTS were eligible for the study.

Informed consent was not obtained due to the retrospective nature of the study. During the data collection period, patients' medical record numbers were retained for reference purposes. Data were deidentified once collection was complete. The research protocol was reviewed and approved by The Johns Hopkins University Institutional Review Board (NA_00029546).

\section{Experimental design}

This study was a retrospective cohort study. Data concerning age at date of visit, race, marital status, parity, use of assisted reproductive technology (ART), family history, and IT uptake were collected from the subjects' medical records. A positive family history was defined as a self-reported history of aneuploidy in a first- or second-degree relative. Date of screen, a priori risk of fetal aneuploidy, and residual risk were collected from the FTS report from NTD labs. When comparing groups with low-elevated residual risk and high-elevated residual risk, we selected $1 / 50$ as a cutoff because prior data indicated that IT uptake is considerably diminished with risk $<1 / 50 .{ }^{21}$ Race was self-reported, and, because of the small sample size of other races in our study population, only white, black/African American, and Asian were used as categories for analysis. We thought it was important to include race as a variable in this study to identify any racial disparities in IT uptake.

\section{First trimester screening}

FTS is performed between 11 and 13 weeks. The screen as performed at the JHPDC uses patient age, serum markers, and ultrasound markers to calculate a probability that a fetus has Down syndrome and a separate probability that it has trisomy 13 or 18 . Serum analysis and risk calculation are performed by NTD labs according to their Ultra-Screen protocol. ${ }^{22}$ They report a detection rate of $95 \%$ at a false-positive rate of $2 \%$ for Down syndrome and a detection rate of $95 \%$ at a $0.3 \%$ falsepositive rate for trisomies 18 and $13.5,23,24$ The calculation of a priori risk takes into account a number of factors, including maternal age and gestational age. ${ }^{25}$ The serum markers used to calculate residual risk are pregnancy-associated plasma protein $\mathrm{A}$ and $\beta$-human chorionic gonadotrophin. The ultrasound markers used are nuchal translucency measurement and nasal bone assessment. Patients with a residual risk $\geq 1 / 300$ are considered screen positive.
Both CVS and amniocentesis are available through Johns Hopkins Hospital, and use of either was considered "invasive testing uptake." Patients are counseled that the procedure-related loss rates are approximately $1 / 175$ for CVS and $1 / 300$ for amniocentesis.

\section{Data analysis}

Risk difference (RD) calculations and linear modeling were used for analysis. $P$ values $<0.05$ were considered significant.

\section{RESULTS}

\section{Participant characteristics}

Within our patient population, the average age was 36 , with $68 \%$ of patients aged 35 years or older (Table 1). Seventy-two percent of the patients were white, $15 \%$ black, $12 \%$ Asian, and $1 \%$ Hispanic. Thirteen percent had a family history of aneuploidy, whereas $87 \%$ did not. Sixty-three percent had living children, whereas $37 \%$ were childless. Data regarding marital status were available for $39 \%$ (66) of the subjects. Of these patients, $77 \%$ were married and $23 \%$ were not. Seven percent had conceived using ART, including intracytoplasmic sperm injection, in vitro fertilization, egg donation, and sperm donation.

All study participants had a positive FTS result. Fifty-four percent had a residual risk of aneuploidy $\geq 1 / 50$, and $46 \%$ had a risk of $<1 / 50$. Eighty-seven percent of subjects had a residual risk that increased from their a priori risk and 13\% had a residual risk that decreased. Seventy percent of patients had elevated risk of trisomy 21 only, $13 \%$ had elevated risk of trisomies 18 and 13 only, and $18 \%$ had elevated risk for both.

\section{Traits that correlate with IT uptake}

Of our cohort of patients with positive FTS, $82 \%$ chose to pursue IT. Several characteristics were determined to have a strong correlation with IT uptake (Table 2 and Fig. 1). Marital status had the strongest correlation, with $87 \%$ uptake among married patients and 53\% among unmarried patients $(\mathrm{RD}=$ $34 \%, P=0.024)$.

A general linear model for relative risk with Gaussian error showed significant interaction between the variables of age and family history, so the two traits were analyzed separately $(P=$ $0.009)$. Among patients with no family history of aneuploidy, $86 \%$ of patients of advanced maternal age ( $\geq 35$ years) chose IT compared with only $69 \%$ of younger patients $(\mathrm{RD}=17 \%$, 0.012). A maternal age of 35 years was selected as the cutoff for the designation of "advanced maternal age" as this age was historically used as an "age screen" cutoff for offering IT. ${ }^{2}$ Among patients younger than 35 years, positive family history correlated strongly with IT uptake: $100 \%$ of patients with a family history of aneuploidy chose IT compared with only $69 \%$ of patients with a negative family history $(\mathrm{RD}=31 \%, 0.050)$.

The residual risk number had a positive correlation with IT uptake, as well. When stratifying patients by residual risk, those groups with somewhat elevated risk of $>1 / 100$ and $1 / 51-1 / 100$ had IT uptake of $75 \%(11 / 35)$ and $76 \%$ (11/34), respectively. Groups with higher residual risks of $1 / 26-1 / 50$ and $\geq 1 / 25$ had IT uptake of $84 \%(27 / 32)$ and $91 \%$ (43/47), respectively. These data indicate that IT uptake increases when risk is $\geq 1 / 50$ and further support the use of $1 / 50$ as a cutoff for analysis of low-elevated risk versus high-elevated risk. Patients with a residual risk of $\geq 1 / 50$ had $89 \%$ uptake, and patients with a postscreen risk of $<1 / 50$ had $76 \%$ uptake $(\mathrm{RD}=13 \%, P=$ 0.046). 
Table 1 Participant characteristics

\begin{tabular}{lr}
\hline Participant characteristics & $n(\%)$ \\
\hline Age & $116(68)$ \\
AMA $(\geq 35)$ & $55(32)$ \\
Non-AMA $(<35)$ & \\
Race &
\end{tabular}

Race

White

Black

Asian

Hispanic

Other

Family history of aneuploidy

Yes

No

Postscreen risk

$$
\geq 1 / 50
$$

$<1 / 50$

Marital status

Married

Unmarried

Not reported

Type of risk

Trisomy 21 only

Trisomy 18/13 only

Trisomy 21 and 18/13

Use of ART

Yes

No

Number of living children

0

$>0$

Number of abortions or miscarriages

0

$>0$

$86(50)$

AMA, advanced maternal age.

Correlation between IT uptake and race was also strong: whites had 90\% uptake, whereas nonwhites had 67\% uptake $(\mathrm{RD}=23 \%, P=0.002)$. Among nonwhites, Asian patients had $71 \%$ uptake, whereas black patients had $60 \%$ uptake. This difference was not statistically significant $(P=0.105)$. Hispanics were not analyzed as a separate group, as only two Hispanic patients were included in our study population.

Also note that IT uptake decreased significantly from the first year of the study $(100 \%$ in 2001$)$ to the last $(56 \%$ in 2009$)$ (Table 3 and Fig. 2). Linear regression yields a slope of $-5 \%$
Table 2 Characteristics correlating with IT uptake

\begin{tabular}{lccc}
\hline Factors & IT uptake & Risk \\
difference & $P$ \\
\hline
\end{tabular}

Maternal age

$$
\begin{aligned}
& \geq 35 \mathrm{yr} \\
& <35 \mathrm{yr}
\end{aligned}
$$

$86 \%(89 / 103)$

$17 \%$

0.012

Race

$$
\text { White }
$$

$69 \%(31 / 45)$

Family history (age <35)

$$
\text { Family history }
$$

$100 \%(10 / 10)$

$31 \%$ 0.050

No family history

$69 \%(31 / 48)$

Residual risk

\begin{tabular}{llll} 
High elevated $(\geq 1 / 50)$ & $89 \%(70 / 92)$ & $13 \%$ & 0.046 \\
Low elevated $(<1 / 50)$ & $76 \%(70 / 79)$ & & \\
Marital status & & & \\
Married & $87 \%(45 / 52)$ & $34 \%$ & 0.024 \\
Unmarried & $53 \%(8 / 15)$ & & \\
\hline
\end{tabular}

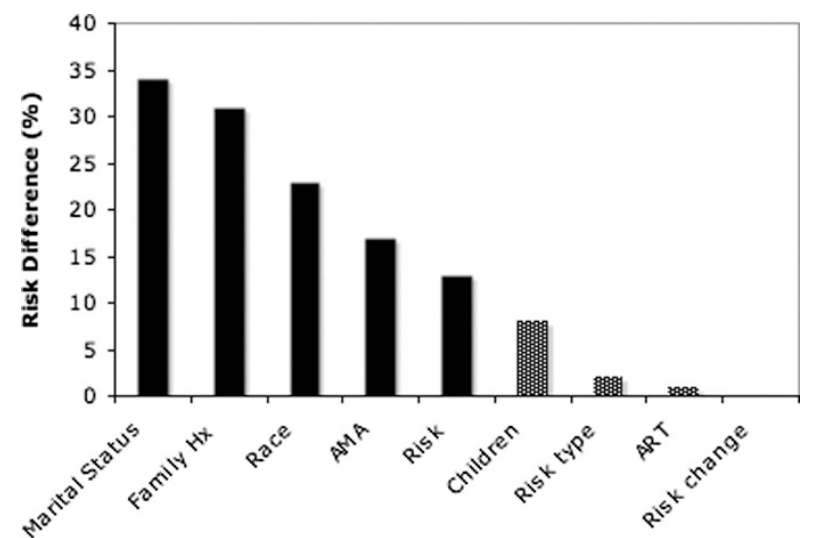

Fig. 1. Comparison of the risk differences for the characteristics studied in relationship to invasive testing uptake. Solid bars represent a correlation with invasive testing uptake, and patterned bars represent no significant correlation. AMA, advanced maternal age; and ART, assisted reproductive technology.

with an $R^{2}$ value of 0.84 , indicating a strong negative correlation between time and IT uptake.

\section{Traits that do not correlate with IT uptake}

Traits that showed no correlation with IT uptake include type of elevated risk, ART use, number of living children, and direction of change from a priori risk (Table 4 and Fig. 1). For risk type, uptake of IT in patients with an elevated risk for trisomy 21 and in patients with an elevated risk for trisomies 18 and 13 was compared. The two groups had IT uptake of $83 \%$ and $85 \%$, respectively $(\mathrm{RD}=2 \%, P=0.41$.) As for ART use, $83 \%$ of patients who had conceived using ART chose IT com- 
Table 3 Invasive testing (IT) uptake by year

\begin{tabular}{lc}
\hline Visit date & IT uptake (\%) \\
\hline 2001 & $1 / 1(100)$ \\
2002 & $3 / 3(100)$ \\
2003 & $5 / 5(100)$ \\
2004 & $19 / 20(95)$ \\
2005 & $32 / 37(86)$ \\
2006 & $23 / 27(85)$ \\
2007 & $28 / 39(72)$ \\
2008 & $24 / 30(80)$ \\
2009 & $5 / 9(56)$ \\
\hline
\end{tabular}

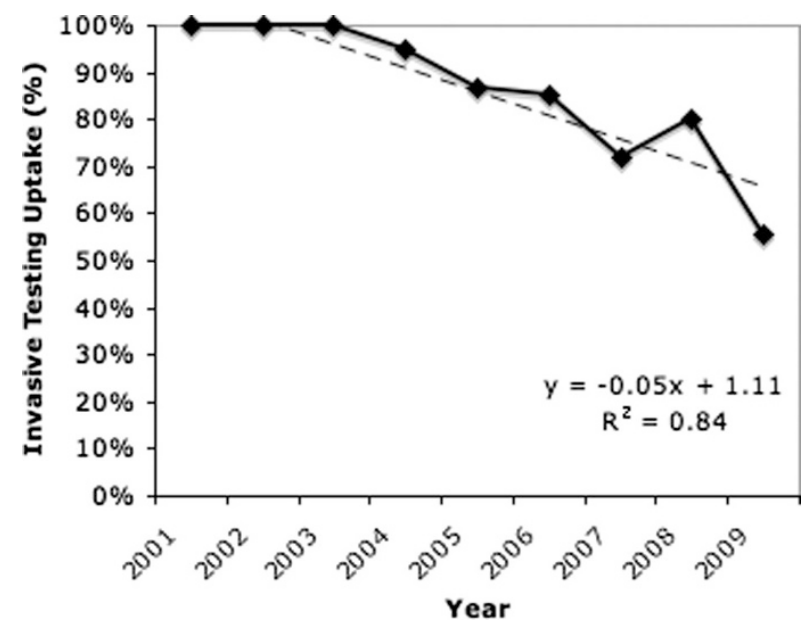

Fig. 2. Percent invasive testing uptake by year. The dashed line represents the linear regression for these data.

pared with $82 \%$ who did not use ART $(\mathrm{RD}=1 \%, P=0.67)$. Patients who had no living children had $77 \%$ uptake, whereas patients with one or more children had $85 \%$ uptake $(\mathrm{RD}=8 \%$, $P=0.16)$. Finally, IT uptake was the same $(82 \%)$ for patients whose residual risk was greater than their a priori risk and for patients whose residual risk was the same or less than their a priori risk $(\mathrm{RD}=0 \%, P=1)$.

\section{DISCUSSION}

This study identified several demographic and historical factors that correlate with the uptake of IT after a positive first trimester aneuploidy screen. Patients with advanced maternal age, white race, married status, positive family history, increased residual risk, and early year of screen were more likely to elect IT. Traits that do not correlate with IT uptake include the type of elevated risk, ART use, number of living children, and direction of change from a priori risk. The decision to undergo IT is complex and unique to each patient. The trends identified in this study invite speculation about common themes in this process and suggest areas of improvement on the part of health care personnel.
Table 4 Characteristics not correlating with IT uptake

\begin{tabular}{llll}
\hline Factor & \multicolumn{1}{c}{ IT uptake } & $\begin{array}{c}\text { Risk } \\
\text { difference }\end{array}$ & $P$ \\
\hline Risk type & & & \\
$\quad$ Trisomy 21 & $83 \%(123 / 149)$ & $2 \%$ & 0.41 \\
$\quad$ Trisomy 18 and 13 & $85 \%(44 / 52)$ & & \\
ART & & & 0.67 \\
$\quad$ ART & $83 \%(10 / 12)$ & $1 \%$ & \\
$\quad$ No ART & $82 \%(130 / 159)$ & & 0.16 \\
Living children & & & \\
$\quad 0$ & $77 \%(49 / 64)$ & $8 \%$ & \\
$>0$ & $85 \%(91 / 107)$ & & \\
Change from a priori risk & & & \\
Increase & $82 \%(116 / 142)$ & $0 \%$ & \\
$\quad$ Decrease or none & $82 \%(18 / 22)$ & & \\
\hline
\end{tabular}

Maternal age was of particular interest for this study. Given that 35 years was formerly used as the cutoff in an "age screen" for IT and the widespread knowledge that older mothers have elevated risk for fetal chromosome abnormalities, we predicted that patients older than 35 years would have significantly higher uptake of IT. After adjusting for significant interaction with family history, our data indeed showed that mothers of advanced maternal age are more likely to opt for IT. The factor, or factors, driving this phenomenon remains unclear. Patients may have a poor understanding of the concept of residual risk and, thus, default to their knowledge that older women are at increased risk. Alternatively, older women may have different value systems that result in their being likely to opt for IT. These patients may be more comfortable with IT if they recall the days before screening was widely available and IT was even more common.

Among patients younger than 35 years, we found a significant association between family history and IT. In fact, all patients younger than 35 years with a family history of fetal aneuploidy elected to undergo IT. These patients may have pursued prenatal diagnosis more aggressively than their peers, believing themselves to be at a higher a priori risk. Alternatively, perhaps their decisions are influenced by personal experience with individuals with chromosome abnormalities.

The change in IT uptake over time is also notable. The first three study years (2001-2003) were found to have 100\% IT uptake among patients with positive FTS results. Uptake decreased at a rate of 5\% per year until the last study year, 2009, when IT uptake was only $56 \%$. Although the first and last years of the study period have fewer patients than the middle years (nine total in 2001-2003 and nine in 2009), the pattern is still impressive. It suggests a changing role of the FTS. Perhaps, patients are using the screen for reassurance despite being unwilling to pursue IT, or perhaps, there is a growing willingness to raise and care for children with disabilities.

The fact that patients with a risk $\geq 1 / 50$ as determined by the FTS had significantly higher IT uptake than patients with a risk $<1 / 50$ is important. It indicates that patients take their numerical risk rather than just their "elevated" risk status into consideration when making decisions about IT. 
Race also emerged as a strong predictor of IT uptake. The reasons for this finding are unclear and may relate to differing values or cultural norms.

Overall, marital status was the strongest predictor of IT uptake. This finding was unanticipated and could be explained by a variety of different factors, including the stability of the parental relationship and the desire or willingness to have more children in the future. Furthermore, in our population, race and/or marital status may be markers for socioeconomic status.

As for traits that did not correlate with higher IT uptake, we were particularly interested in the effect of the type of elevated risk (trisomy 21 or trisomies 18 and 13) on IT uptake because of the different prognoses of the trisomies. Compared with trisomy 18 or 13 , Down syndrome is less lethal at a young age and also is more present in the public awareness. Patients with trisomy 13 have a high rate of early embryonic death (98-99\%). Those who are live born rarely $(5 \%)$ survive the first 6 months of life and, if they do, suffer from severe intellectual disability, seizures, and failure to thrive. ${ }^{26}$ Among patients born with trisomy 18 , only $5-10 \%$ survive the first year of life, and those who do have similar severe intellectual and physical impairment. ${ }^{27}$ Patients born with trisomy 21, on the other hand, may have an IQ of up to 70 and have a life expectancy of 49 years. ${ }^{28,29}$ We hypothesized that patients may be more willing to consider pregnancy termination and, therefore, would be more likely to pursue IT if they were told they had a high risk for trisomies 18 and 13 compared with trisomy 21. Our data suggest, however, that the type of elevated risk does not impact patients' decisions about IT in a significant way.

ART use was also of interest, as prior studies have mixed results regarding IT uptake after ART use and second trimester screening. Our analysis showed no correlation between ART use and IT uptake. It should be noted, however, that only 12 of our 172 study participants used ART.

IT among patients whose residual risk was greater than their a priori risk was the same as uptake in patients whose residual risk was less than or equal to their a priori risk. The import of this finding is unclear, particularly because some providers may be more explicit in their comparison of residual risk to a priori risk than others.

Although all patients received similar counseling through the JHPDC before IT, there may be substantial variation in the amount and quality of counseling received before the FTS. A 1995 study revealed that many providers present prenatal aneuploidy screening as routine care rather than one requiring a decision on the part of the patient. ${ }^{30}$ Furthermore, 1994 survey found that $25 \%$ of primary obstetricians regularly offered the screen as part of routine prenatal care without any explanation of its primary purpose. ${ }^{31}$ Although these studies were both conducted in the United Kingdom with the second trimester screen, similar practices are likely at play in our population today. Some patients referred to the JHPDC may have been receiving information about the risks and benefits of IT for the first time. It is possible that some patients with a positive FTS who declined IT would never even have considered IT and, therefore, may have been poor FTS candidates in the first place.

\section{Future directions and conclusions}

This study reveals patterns of IT uptake in patients with a positive FTS. The reasons for these patterns are as yet unclear and implicate numerous avenues for further study. For example, additional demographic information could be collected. We hypothesize that socioeconomic status may be a confounder in the relationship between maternal age, marital status, and/or race and IT uptake. Data on household income, educational level, and insurance status could help clarify these relationships. Additionally, patients' understanding of the FTS before and after counseling could be gauged using the Multidimensional Measure of Informed Choice, described earlier in the text. This assessment has revealed striking deficiencies in knowledge in all the populations for which data have been published and, notably, has not yet been studied in the United States. These results could be compared with demographic factors to determine whether any of the characteristics we identified as predictive for IT uptake are associated with poor information about the test.

This study identifies groups at high risk of declining IT after a positive first trimester screen, including patients who are unmarried, young, and nonwhite. These patients may benefit from additional counseling both before administration of the FTS and after receipt of the results to ensure that they are making informed decisions.

\section{ACKNOWLEDGMENTS}

This research was supported by The Johns Hopkins University School of Medicine Dean's Fund for student research and by Grant number UL1 RR 025005 from the National Center supported statistical analysis for Research Resources (NCRR), a component of the National Institutes of Health (NIH), and NIH Roadmap for Medical Research.

\section{REFERENCES}

1. Jackson LG, Zachary JM, Fowler SE, et al. A randomized comparison of transcervical and transabdominal chorionic-villus sampling. The U.S. National Institute of Child Health and Human Development Chorionic-Villus Sampling and Amniocentesis Study Group. N Engl J Med 1992;327:594598.

2. Ball RH, Caughey AB, Malone FD, et al. First- and second- trimester evaluation of risk for Down syndrome. Obstet Gynecol 2007;110:10-17.

3. Benn PA, Fang M, Egan JF. Trends in the use of second trimester maternal serum screening from 1991 to 2003. Genet Med 2005;7:328-331.

4. Cicero S, Spencer K, Nicolaides KH, Avgidou K, Faiola S. Maternal serum biochemistry at 11-13(+6) weeks in relation to the presence or absence of the fetal nasal bone on ultrasonography in chromosomally abnormal fetuses: an updated analysis of integrated ultrasound and biochemical screening. Prenat Diagn 2005;42:977-983.

5. Nicolaides KH. The $11-13^{+6}$ weeks scan. London: Fetal Medicine Foundation, 2004. In: Gelehrter TD, Collins FS, Ginsburg D, editors. Principles of medical genetics, 2nd ed. Baltimore: Williams \& Wilkins, 1998:11.

6. Gourounti K, Sandall J. Do pregnant women in Greece make informed choices about antenatal screening for Down's syndrome? A questionnaire survey. Midwifery 2008;24:153-162.

7. Marini T, Sullivan J, Naeem R. Decisions about amniocentesis by advanced maternal age patients following maternal serum screening may not always correlate clinically with screening results: need for improvement in informed consent process. Am J Med Genet 2002;109:171-175.

8. Michie S, Smith D, Marteau TM. Prenatal tests: how are women deciding? Prenat Diagn 1999;19:743-748.

9. Marteau TM, Dormandy E, Michie S. A measure of informed choice. Health Expect 2001;4:99-108.

10. Fransen MP, Essink-Bot ML, Vogel I, et al. Ethnic differences in informed decision-making about prenatal screening for Down's syndrome. J Epidemiol Community Health 2010;64:262-268

11. Rowe HJ, Fisher JR, Quinlivan JA. Are pregnant Australian women well informed about prenatal genetic screening? A systematic investigation using the multidimensional measure of informed choice. Aust $N Z$ J Obstet Gynaecol 2006;46:433-439.

12. Abu-Musa AA, Nassar AH, Usta IM. Attitude of women with IVF and spontaneous pregnancies towards prenatal screening. Hum Reprod 2008;23: 2438-2443.

13. Mueller VM, Huang T, Summers AM, Winsor SH. The influence of risk estimates obtained from maternal serum screening on amniocentesis rates. Prenat Diagn 2005;25:1253-1257.

14. Nicolaides KH, Chervenak FA, McCullough LB, Avgidou K, Papageorghiou A. Evidence-based obstetric ethics and informed decision-making by pregnant women about invasive diagnosis after first-trimester assessment of risk for trisomy 21. Am J Obstet Gynecol 2005;193:322-326.

15. Rice JD, McIntosh SF, Halstead AC. Second-trimester maternal serum 
screening for Down syndrome in in vitro fertilization pregnancies. Prenat Diagn 2005;25:234-238.

16. Ilgin-Ruhi H, Yürür-Kutlay N, Tükün A, Bökesoy I. The role of genetic counseling on decisions of pregnant women aged 35 years or over regarding amniocentesis in Turkey. Eur J Med Genet 2005;48:13-19.

17. Priest JH, FitzGerald JM, Haag MM, Streets K, Vanisko M, Johnson JP. Acceptance of amniocentesis by women in the state of Montana (USA) who are screen positive for Down's syndrome. J Med Screen 1998;5:178-182.

18. Geipel A, Berg C, Katalinic A, et al. Different preferences for prenatal diagnosis in pregnancies following assisted reproduction versus spontaneous conception. Reprod Biomed Online 2004;8:119-124.

19. Caughey AB, Musci TJ, Belluomini J, Main D, Otto C, Goldberg J. Nuchal translucency screening: how do women actually utilize the results? Prenat Diagn 2007;27:119-123

20. Wray AM, Ghidini A, Alvis C, Hodor J, Landy HJ, Poggi SH. The impact of first-trimester screening on AMA patients' uptake of invasive testing. Prenat Diagn 2005;25:350-353.

21. Wagner D, Pargas C, Donnenfeld AE. Moderately increased risks of Down's syndrome $(1 / 51-1 / 270)$ identified on first trimester sequential screening: what do patients do with this information? J Med Screen 2010;17:4-7.

22. NTD Labs, 2009. Available at: http://www.ntdlabs.com/protocols/ultra screen_protocol.php. Accessed March 2, 2010.

23. Perni SC, Predanic M, Kalish RB, Chervenak FA, Chasen ST. Clinical use of first-trimester aneuploidy screening in a United States population can replicate data from clinical trials. Am J Obstet Gynecol 2006;194:127-130.

24. Spencer K, Nicolaides KH. A first trimester trisomy 13/trisomy 18 risk algorithm combining fetal nuchal translucency thickness, maternal serum free Fß-hCG and PAPP-A. Prenat Diagn 2002;22:877-879.

25. Palomaki GE, Lee JE, Canick JA, et al. Technical standards and guidelines: prenatal screening for Down syndrome that includes first-trimester biochemistry and/or ultrasound measurements. Genet Med 2009;11:669-681.

26. Tolmie JL. Down syndrome and other autosomal trisomies. In: Rimoin DL, Connor JM, Pyeritz RE, editors. Emery and Rimoin's principles and practice of medical genetics, 3rd ed. New York: Churchill Livingston, 1996:925.

27. Jones KL. Smith's recognizable patterns of human malformation, 6th ed. Philadelphia: Elsevier Saunders, 2006

28. American Academy of Pediatrics, Committee on Genetics. American Academy of Pediatrics: health supervision for children with Down syndrome. Pediatrics 2001;107:442-449.

29. Yang Q, Rasmussen SA, Friedman JM. Mortality associated with Down's syndrome in the USA from 1983 to 1997: a population-based study. Lancet 2002;359:1019-1025.

30. Smith DK, Shaw RW, Slack J, Marteau TM. Training obstetricians and midwives to present screening tests: evaluation of two brief interventions. Prenat Diagn 1995;15:317-324.

31. Green JM. Serum screening for Down's syndrome: experiences of obstetricians in England and Wales. BMJ 1994;309:769-772. 